International Journal of Pure and Applied Mathematics

Volume 91 No. 1 2014, 1-12

ISSN: $1311-8080$ (printed version); ISSN: 1314-3395 (on-line version)

url: http://www.ijpam.eu

doi: http://dx.doi.org/10.12732/ijpam.v91i1.1

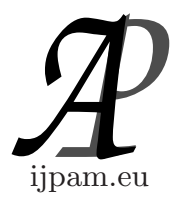

\title{
A FINITE DIFFERENCE SCHEME FOR MAGNETO HYDRO DYNAMIC FLUID FLOW IN SLIDER BEARING
}

\author{
M.M. Wafula ${ }^{1}$, J.K. Kwanza ${ }^{2}$, J.A. Okello ${ }^{3}$ \\ 1,2,3 Department of Pure and Applied Mathematics \\ Jomo Kenyatta University of Agriculture and Technology \\ P.O. Box 62000-00200, Nairobi, KENYA
}

\begin{abstract}
A mathematical model of magneto hydro dynamic fluid flow in a slider bearing is presented. A generalized two dimensional Reynolds-type equation is derived using the equations of motion. The equation is solved numerically using finite difference scheme which involves the conjugate Gradient method. The pressure is used to evaluate the bearing characteristics such as load carrying capacity and friction parameter. The results are presented graphically. It is found that increased magnetic field leads to increased friction.
\end{abstract}

Key Words: magnetohydrodynamic, Reynolds equation, magnetic field, aspect ratio, friction

\section{Introduction}

Lubrication occurs in engines and machines to reduce friction between the moving plates. Slider bearings are encountered in engineering applications and other fields of science.Various kinds of fluids are used as lubricants. Recently the use of electrically conducting fluid has received considerable attention.

Several researchers have investigated the Magnetohydrodynamic (MHD)

Received: October 13, 2012

(C) 2014 Academic Publications, Ltd.

$\S_{\text {Correspondence author }}$ url: www.acadpubl.eu 
characteristics of bearings with electrically conducting fluids in the presence of magnetic fields. By supplying electrical power from an external source, Huges [1] studied the effect of field geometries on the MHD Rayleigh step bearing.In the study of MHD one-dimensional inclined plane slider bearings by Synder [2], load enhancement was predicted with liquid metal lubricants in the presence of a magnetic field. Investigations on MHD wide slider bearing were carried out by Agrawal [3].He found that inertia effects increased the load carrying capacity for small Hartman numbers.

Chouet al [4] studied the effects of externally applied magnetic fields on the squeeze film characteristics between a sphere and a flat plate with an externally conducting fluid. Comparing with the classical non-conducting lubricant case, the effects of externally applied magnetic fields characterized by Hartman number provide an enhancement to the magneto hydrodynamic load carrying capacity and lengthen the response time, especially for large values of Hartman number. Lin and Hung [5] analyzed dynamic characteristics for wider slider bearing with an exponential film profile taking into account the bearing squeezing action. They concluded that both the steady state performance and the dynamic characteristics are significantly affected by the inlet outlet film ration of the slider bearing. Lin and $\mathrm{Lu}[6]$ investigated the dynamic characteristics of a wide exponential shaped slider bearing with an electrically conducting fluid in the presence of a transverse magnetic field on the basis of the magneto hydrodynamic thin film lubrication. Despite all these investigations, the Hydromagnetic and aspect ratio effect on friction and the load carrying capacity has received little attention. Hence the main objective of this study is to investigate the effects of the magnetic field and aspect ratio on Friction and load carrying capacity of a slider bearing using a finite difference scheme.

\section{Mathematical Analysis}

An electrically conducting fluid flows between two plates of which the upper plate is stationary and the lower plate moves with velocity $U$. The bearing is subjected to a transverse magnetic field $B_{o}$ as shown (Figure 1).The film wedge generates a hydrodynamic pressure field that supports an applied load.

The film thickens $(h)$ has a linear taper along the direction of the surface velocity $\mathrm{u}$.

$$
h=h_{0}-\left(\frac{h_{0}-h_{L}}{L}\right) x .
$$

The length $L$ is in the $\mathrm{x}$-direction and width $\mathrm{B}$ in z-direction. The flow 


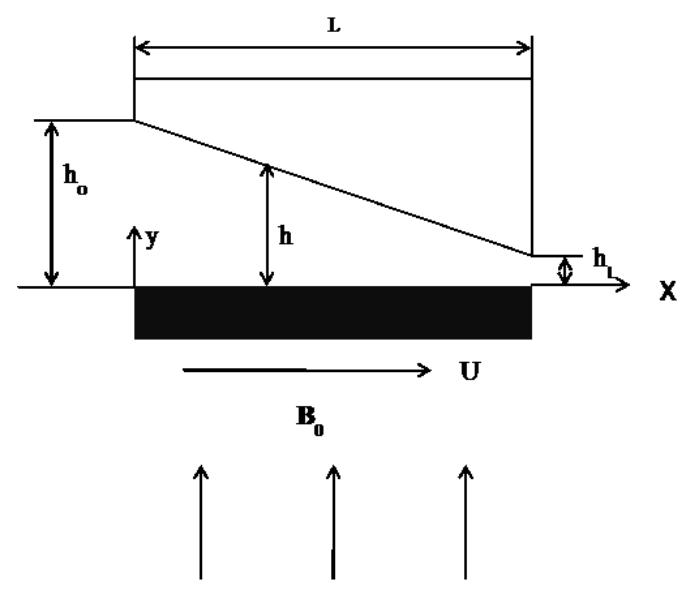

Figure 1: Slider bearing in the presence of a uniform transverse magnetic field

of the fluid is at steady state, the density does not change with time. The continuity equation and momentum equations governing this flow are:

$$
\begin{gathered}
\frac{\partial u}{\partial x}+\frac{\partial v}{\partial y}+\frac{\partial w}{\partial z}=0 \\
\frac{\partial P}{\partial x}=\mu \frac{\partial^{2} u}{\partial y^{2}}-\sigma B_{0}\left(E_{Z}+\mu B_{0}\right), \\
\frac{\partial P}{\partial y}=0, \\
\frac{\partial P}{\partial z}=\mu \frac{\partial^{2} w}{\partial y^{2}}-\sigma B_{0}\left(E_{X}+\mu B_{0}\right) .
\end{gathered}
$$

The boundary conditions at the two surfaces of the bearing are

$$
\begin{gathered}
u(x, 0, z)=U, v(x, 0, z)=0, w(x, 0, z)=0, \\
u(x, h, z)=0, v(x, h, z)=0, w(x, h, z)=0 .
\end{gathered}
$$

By applying the above boundary conditions the velocity component $u$ and $w$ are solved from equations (3) and (5) respectively

$$
u=U\left[\cosh \left(\frac{M y}{h_{L}}\right)-\operatorname{coth}\left(\frac{M h}{h_{L}}\right) \sinh \left(\frac{M y}{h_{L}}\right)\right]+\left(\frac{h_{L}^{2}}{\mu M^{2}} \frac{\partial P}{\partial x}+\frac{E_{Z}}{B_{0}}\right)
$$




$$
\begin{array}{r}
{\left[\cosh \left(\frac{M y}{h_{L}}\right)+\frac{1-\cosh \left(\frac{M h}{h_{L}}\right)}{\sinh \left(\frac{M h}{h_{L}}\right)} \sinh \left(\frac{M h}{h_{L}}\right)-1\right],} \\
w=\left(\frac{h_{L}^{2}}{\mu M^{2}} \frac{\partial P}{\partial z}+\frac{E_{x}}{B_{0}}\right) \\
{\left[\cosh \left(\frac{M y}{h_{L}}\right)+\frac{1-\cosh \left(\frac{M h}{h_{L}}\right)}{\sinh \left(\frac{M h}{h_{L}}\right)} \sinh \left(\frac{M h}{h_{L}}\right)-1\right] .}
\end{array}
$$

Here M denotes the Hartmann number

$$
B_{0} h_{L}\left(\frac{\sigma}{\mu}\right)^{\frac{1}{2}} .
$$

The bearing surfaces are perfect insulators and there is no current external to the film, the electric field may be approximated by requiring the net current flow to be zero

$$
\begin{gathered}
\int_{y=0}^{y=h}\left(E_{Z}+B_{0} u\right) d y=0 \\
\int_{y=0}^{y=h}\left(E_{x}-B_{0} w\right) d y=0 .
\end{gathered}
$$

Substituting for $\mathrm{u}$ and $\mathrm{w}$ in equations (10)and (11) and performing the integration, the velocity component $u$ and $w$ becomes

$$
\begin{gathered}
u=\frac{U}{2}\left[1-\frac{\sinh \left(\frac{M y}{h_{L}}\right)-\sinh \left(\frac{M h}{h_{L}}\right)}{\sinh \left(\frac{M h}{h_{L}}\right)}\right]+\frac{h_{L} h}{2 \mu M} \frac{\partial p}{\partial x} \operatorname{coth}\left(\frac{0.5 M h}{h_{L}}\right) \\
{\left[\frac{\sinh \left(\frac{M h}{h_{L}}\right)+\sinh \left(\frac{M h}{h_{L}}-\frac{M y}{h_{L}}\right)}{\sinh \left(\frac{M h}{h_{L}}\right)}-1\right],} \\
w=\frac{h_{L} h}{2 \mu M} \frac{\partial p}{\partial z} \operatorname{coth}\left(\frac{0.5 M h}{h_{L}}\right)\left[\frac{\sinh \left(\frac{M h}{h_{L}}\right)+\sinh \left(\frac{M h}{h_{L}}-\frac{M y}{h_{L}}\right)}{\sinh \left(\frac{M h}{h_{L}}\right)}-1\right]
\end{gathered}
$$




\subsection{MHD Reynolds Equation}

Integrating the continuity equation (2) across the film we have

$$
\int_{y=0}^{y=h} \frac{\partial u}{\partial x} \partial y+\int_{y=0}^{y=h} \frac{\partial v}{\partial y} \partial y+\int_{y=0}^{y=h} \frac{\partial w}{\partial z} \partial y=0 .
$$

Performing the integration by using the boundary conditions (6), and substituting the velocity components in the above integral, the general MHD Reynolds-type equation is derived as

$$
\frac{\partial}{\partial x}\left[a\left(M, h, h_{L}\right) \frac{\partial P}{\partial x}\right]+\frac{\partial}{\partial z}\left[a\left(M, h, h_{L}\right) \frac{\partial P}{\partial z}\right]=\frac{\mu U}{2} \frac{\partial h}{\partial x},
$$

where the function is $a\left(M, h, h_{L}\right)$ is defined as follows

$$
a\left(M, h, h_{L}\right)=\frac{h_{L}^{2} h}{2 M^{2}}\left[\frac{M h}{h_{L}} \operatorname{coth}\left(\frac{M h}{2 h_{L}}\right)-2\right] .
$$

Introducing the dimensionless quantities:

$$
x^{*}=\frac{X}{L}, \quad z^{*}=\frac{B}{L}, \quad h^{*}=\frac{h}{h_{L}}=\alpha-(\alpha-1) x^{*}, \quad \alpha \frac{h_{o}}{h_{l}} .
$$

Using these quantities, equation (15) yields

$$
\frac{\partial}{\partial x^{*}}\left[A\left(M, h^{*}\right) \frac{\partial P^{*}}{\partial x^{*}}\right]+\frac{1}{\beta^{2}} \frac{\partial}{\partial z^{*}}\left[A\left(M, h^{*}\right) \frac{\partial P^{*}}{\partial z^{*}}\right]=\frac{1}{2} \frac{d h^{*}}{d x^{*}}
$$

Equation (17) is a dimensionless Reynolds type equation where

$$
\begin{aligned}
& A\left(M, h^{*}\right)=\frac{h^{*}}{2 M^{2}}\left[M h^{*} \operatorname{coth}\left(\frac{M h^{*}}{2}\right)-2\right] \text { and } \\
& \beta=\frac{B}{L} \text { represent the aspect ratio. }
\end{aligned}
$$

As M approaches zero, equation (17)reduces to the classical Reynolds-type equation Hamrock[7]. It is assumed that the lubricant is enough to flood the bearing, and the cavitation effect is neglected. Then the pressure boundary conditions are given by

$$
\begin{aligned}
& p^{*}=0 \text { at } x^{*}=0 \text { and } p^{*}=0 \text { at } x^{*}=1, \\
& p^{*}=0 \text { at } z^{*}=0 \text { and } p^{*}=0 \text { at } z^{*}=1 .
\end{aligned}
$$




\subsection{Solutions}

The dimensionless MHD Reynolds-type equation with conditions (19) and (20) is now solved numerically by using finite difference schemes. Using the centraldifference approximation, the dimensionless MHD Reynolds-type equation is written in a finite difference format as

$$
\gamma_{0} p_{i, j}^{*}+\gamma_{1} p_{i+1, j}^{*}+\gamma_{2} p_{i-1, j}^{*}+\gamma_{3} p_{i, j+1}^{*}+\gamma_{4} p_{i, j-1}^{*}=\delta,
$$

where:

$$
\begin{gathered}
\gamma_{0}=-\beta^{2} b^{2}\left(A_{i+\frac{1}{2}, j}+A_{i-\frac{1}{2}, j}\right)+A_{i, j+\frac{1}{2}}+A_{i, j+\frac{1}{2}}, \\
\gamma_{1}=\beta^{2} b^{2} A_{i+\frac{1}{2}, j}, \\
\gamma_{2}=\beta^{2} b^{2} A_{i-\frac{1}{2}, j}, \\
\gamma_{3}=A_{i, j+\frac{1}{2}} \\
\gamma_{4}=A_{i, j-\frac{1}{2}} \\
\delta=-0.5 \beta^{2} b^{2}\left(\triangle x^{*}\right)^{2}(\alpha-1), \\
b=\frac{\triangle z^{*}}{\triangle x^{*}} .
\end{gathered}
$$

Equation (21) with boundary conditions (19)and (20) is numerically solved for pressure using the Conjugate Gradient Method (CGM). Based on a threeterm recurrence, the CGM is an unconstrained optimization technique in which the search directions are conjugate, Decker et al, [8]

The finite termination property implies that this method is guaranteed to terminate after a finite number of steps. Let the residual vector $r_{i, j}$ be

$$
r_{i, j}=\gamma_{0} p_{i, j}^{*}+\gamma_{1} p_{i+1, j}^{*}+\gamma_{2} p_{i-1, j}^{*}+\gamma_{3} p_{i, j+1}^{*}+\gamma_{4} p_{i, j-1}^{*}-\delta .
$$

Then the pressure values have converged according to the following convergent criteria

$$
\left[\frac{\left|\sum r_{i, j}^{k+1} r_{j, i}^{k+1}\right|}{\left|\sum r_{i, j}^{k} r_{j, i}^{k}\right|}\right]^{\frac{1}{2}}<10^{-3}
$$




\subsection{MHD Bearing Characteristics}

With the film pressure obtained, the hydro-dynamic squeeze film force can be evaluated. The MHD load-carrying capacity is calculated by integrating the pressure over the film region

$$
W=\int_{x=0}^{x=L} \int_{z=0}^{z=B} p d z d x .
$$

Let us introduce the dimensionless quantity

$$
W^{*}=\frac{W h_{L}^{2}}{\mu U L^{2} B} .
$$

The dimensionless load-carrying capacity can be expressed in a difference form

$$
W^{*}=\triangle x^{*} \triangle z^{*} \sum_{i=0}^{m} \sum_{j=0}^{n} p_{i, j}^{*} .
$$

The shear stress which the film exerts on the lower surface is

$$
\tau_{s}=\left.\mu \frac{\partial u}{\partial y}\right|_{y=0}
$$

Expressed in a dimensionless form one has

$$
\tau_{s}^{*}=-\frac{1}{2}\left[M \operatorname{coth}\left(0.5 M h^{*}\right)+h^{*} \frac{\partial p^{*}}{\partial x^{*}}\right] .
$$

The friction force acting on the slider is calculated by integrating the shear stress over the film region

$$
F=\int_{z=0}^{z=B} \int_{x=0}^{x=L} \tau_{s} d x d z .
$$

Integration, we obtain in dimension less form the result,

$$
F^{*}=\frac{1}{\alpha-1} \ln \frac{\sinh (0.5 M)}{\sinh (0.5 \alpha M)}-\frac{(\alpha-1) W^{*}}{2},
$$

and the coefficient of friction is

$$
f=\frac{F}{W} .
$$

Using non-dimensional quantities, the friction parameter becomes,

$$
C_{f}^{*}=f \cdot \frac{L}{h_{L}}=\frac{F^{*}}{W^{*}} .
$$




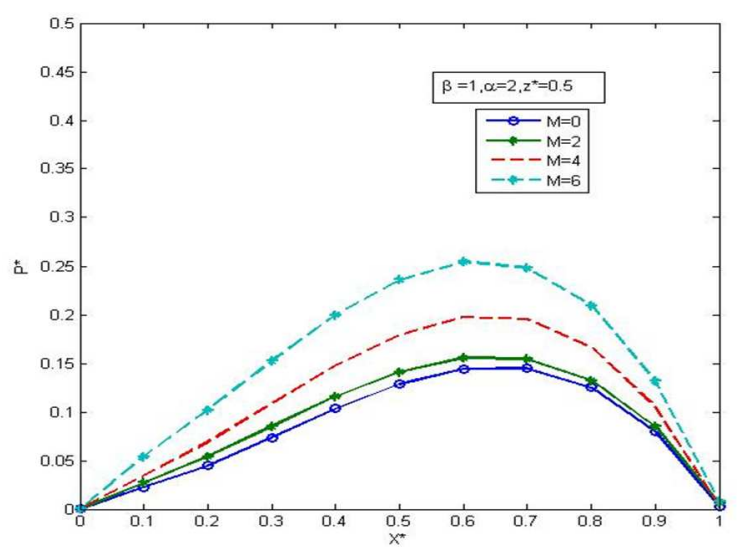

Figure 2: Dimensionless Film pressure in the mid-plane with $\beta=1$ and $\alpha=2$ for different $\mathrm{M}$

\section{Discussion of the Results}

The film pressure, the load carrying capacity, $\mathrm{W}^{*}$ and the friction $\mathrm{Cf}^{*}$ are obtained for various values of the parameters, $\beta, \alpha$ and $\mathrm{M}$.

These symbols are defined in the nomenclature.

Figure 2 represents the dimensionless film pressure $\mathrm{P}^{*}$ as a function of dimensionless coordinates $\mathrm{X}^{*}$ in the mid-plane $(z=0.5)$ with $\beta=1, \alpha=2$ and different values of the Hartman number $M$.The value $\mathrm{M}=0$ represents the case when the bearing is not subjected to a magnetic field.

It is noted that the film pressure increases with increase in the value of the magnetic field parameter $\mathrm{M}$.

Figure 3 Shows the dimensionless film pressure as a function of the Hartman number at film thickness ratio $\alpha=2$ for different values of the aspect ratio $\beta$. It is seen that as the aspect ratio increases the film pressure $\mathrm{P}^{*}$ increases with increased Hartman number.

Figure 5 shows how the friction parameter $C f *$ varies with the Hartmann number $\mathrm{M}$ at $\alpha=2$ for different values of aspect ratio. It is noted increased magnetic field leads o increased friction and the higher the aspect ratio, the more the friction. 


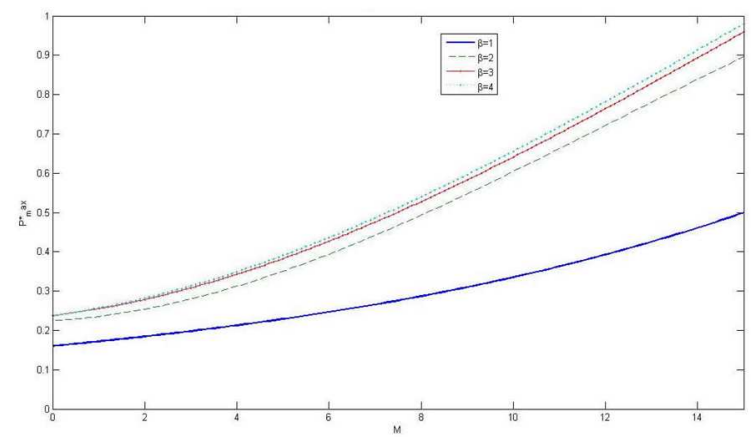

Figure 3: Variation of Dimensionless pressure $\mathrm{P}^{*}$ with Hartman Number $\mathrm{M}$ at $\alpha=2$ for different values of $\beta$.

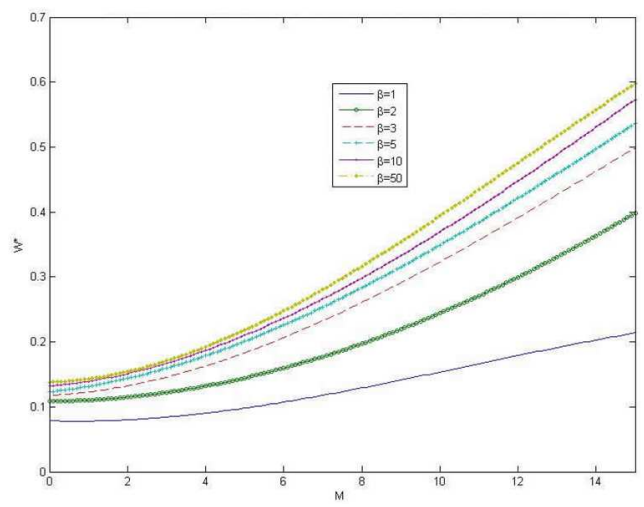

Figure 4: Variation of Dimensionless load carrying capacity $\mathrm{W}^{*}$ with the Hartman number $\mathrm{M}$ at $\alpha=2$ for different values of $\beta$.

\section{Validation of Results}

Our results were compared with those of Patel et al [9] who used analytical method in their investigation. They found that increase in aspect ratio led to increased load carrying capacity. This result, coincide with our findings in the present study. However they did not consider the effect of the Magnetic field on friction which we have considered in our investigations. It is found that Magnetic field enhances friction in the bearing. 


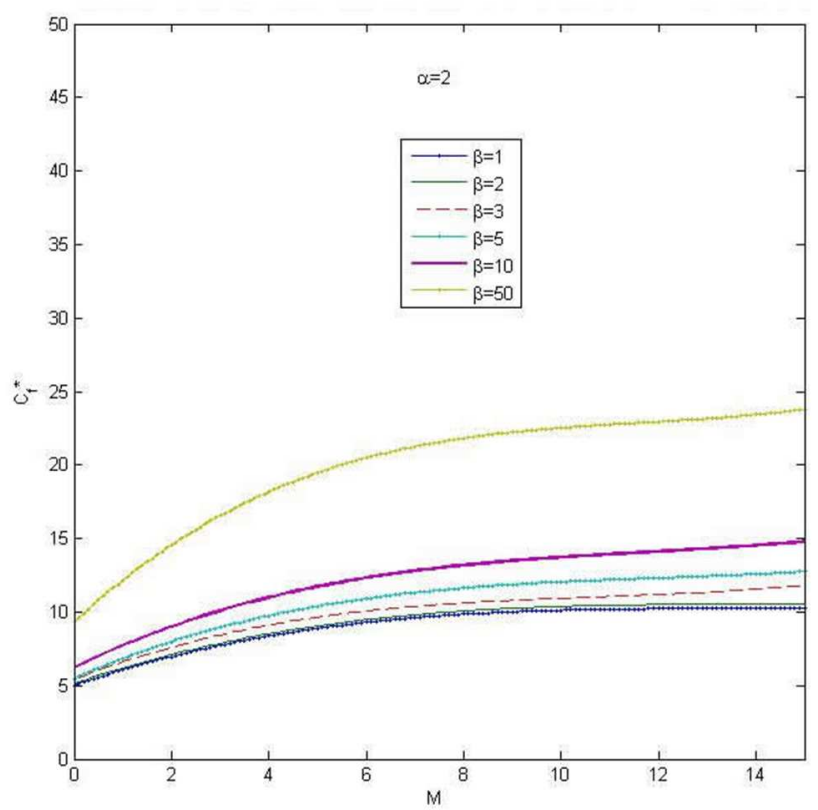

Figure 5: Variation of Dimensionless friction parameter $\mathrm{Cf}^{*}$ with the Hartman number $\mathrm{M}$ at $\alpha=2$ for different values of $\beta$

\section{Conclusion}

From the discussion of results, it is noted that the magnetic field increases the film pressure. Thus the load carrying capacity of the bearing is enhanced by the application of the magnetic field.In presence of magnetic field, increase in aspect ratio leads to increased pressure as well as friction in the bearing.

\section{References}

[1] W.F. Huges, The magneto hydrodynamic finite step slider bearing, ASME Journal of Basic Engineering, 85 (1963), 129-136.

[2] T.W. Synder, The non-linear hydrodynamic slider bearing, ASME Journal of Lubrication Technology, 62 (1963), 52-54.

[3] V.K. Agrawal, Inertia effects in hydro magnetic inclined slider bearing, Japanese Journal of Applied Physics, 9 (1970), 820-824. 
[4] T.L. Chou, J.W. Lai, J.R. Lin, Magneto hydrodynamic squeeze film characteristics between a sphere and a plane surface, Journal of Marine Science and Technology, 11 (2003), 174-178.

[5] J.R. Lin, C. R.Hung, Analysis of dynamic characteristics for wide slider bearing with exponential film profile, Journal of Marine Science and Technology, 12 (2004), 217-221.

[6] J.R. Lin, R.F. Lu, Dynamic characteristics for magneto hydrodynamic wide slider bearing with an exponential film profile, Journal of Marine Science and Technology, 18 (2010), 268-276.

[7] B.J. Hamrock, Fundamentals of Fluid Film Lubrication, New York, McGraw-Hill, 1994.

[8] I.C. Decker, D.M. Falcao, E. Kaszkurewicz, Parallel implementation of a power system dynamic simulation methodology using the conjugate gradient method, Trans. On Power Systems, 7 (1992), 458-465.

[9] R.M. Patel, G.M. Deheri, P.A. Vadher, Magnetic fluid based squeeze film between annular plates and transverse surface roughness effect, ANNALS International Journal of Engineering, 8 (2010), 51-55.

\section{A. Nomenclature}

$\alpha$ Inlet-outlet film ratio;

$\beta$ Aspect ratio;

$\mu$ Fluid dynamic viscosity;

$\nabla \quad$ Gradient operator $\left[=i \frac{\partial}{\partial x}+j \frac{\partial}{\partial y}+k \frac{\partial}{\partial z}\right]$;

$\rho \quad$ Fluid density, $\mathrm{kg}^{-3}$;

$\sigma \quad$ Fluid electrical conductivity, $\omega^{-1} m^{-1}$;

$A$ Dimensionless function;

$B_{0} \quad$ Applied magnetic field , $\mathrm{Wbm}^{-2}$;

$C_{f}$ Friction parameter, N;

$D$ Electric displacement vector, $\mathrm{cm}-2$;

$D / D t \quad$ Material derivative;

$E \quad$ Electric field intensity vector, $v m-1$; 
$E_{x}, E_{z} \quad$ Electric field in x-direction and z-directionm,vm-1;

$f$ Friction coefficient;

$F^{*}$ Dimensionless friction force;

$g$ Gravitational field vector, $\mathrm{ms}^{-2}$;

$H \quad$ Magnetic field intensity vector, $w b m-2$;

$h \quad$ Film thickness,m;

$h^{*}$ Dimensionless film thickness;

$h_{0} \quad$ Film thickness at the inlet, $\mathrm{m}$;

$h_{l}$ Film thickness at the outlet, m;

$J \quad$ Current density vector, $A m-2$;

$L \quad$ Length of bearing,m;

$M$ Hartman number;

$p^{*}$ Dimensionless film pressure;

$U$ Sliding velocity, $m s^{-1}$;

$u$ Velocity components in $\mathrm{x}$-directions;

$u, v, w$ Velocity components in $\mathrm{x}-, \mathrm{y}-, \mathrm{z}$-directions;

$W \quad$ Load carrying capacity;

$W^{*}$ Dimensionless load carrying capacity, kg;

$x, y, z$ Rectangular coordinates, $\mathrm{m}$;

$x^{*}, z^{*}$ Dimensionless coordinates;

$\mathrm{B}$ Width of the bearing, $\mathrm{m}$. 